\title{
The interphase model applied to the analysis of masonry structures
}

\author{
Giuseppe Fileccia Scimemi, Giuseppe Giambanco*, Antonino Spada \\ Department of Civil, Environmental, Aerospace Engineering and Materials (DICAM), University of Palermo, Viale delle Scienze Ed. 8, \\ 90128 Palermo, Italy
}

Received 24 April 2014; received in revised form 14 June 2014; accepted 16 June 2014

Available online 24 June 2014

\begin{abstract}
Masonry material presents a mechanical response strongly dependent on the static and kinematic phenomena occurring in the constituents and at their joints. At the mesoscopic level the interaction between the units is simulated by means of specific mechanical devices such as the zero thickness interface model where the contact tractions and the displacement discontinuities are the primary static and kinematic variables respectively.

In many cases the joint response depends also on internal stresses and strains within the interface layer adjacent to the joint interfaces. The introduction of internal stresses and strains leads to the formulation of the interphase model, a sort of enhanced zerothickness interface. With the term interphase we shall mean a layer separated by two physical interfaces from the bulk material or a multilayer structure with varying properties and several interfaces.

Adopting the interphase concept, different failure conditions can be introduced for the physical interfaces and for the joint material. In the present work the interphase constitutive laws, taking into account the joint stiffness degradation and the onset of irreversible displacements, are derived in a thermodynamically consistent manner assuming an appropriate form of the Helmholtz free energy, function of the internal and contact joint strains and of other internal variables which regulate the evolution of the non-linear phenomena.
\end{abstract}

The interphase model has been implemented in an open-source research-oriented finite element analysis program for 2D applications.

(C) 2014 Published by Elsevier B.V.

Keywords: Interphase; Damage; Plasticity; Masonry

\section{Introduction}

Two earthquakes have recently stricken the Italian regions of Emilia (2012) and Abruzzo (2009) providing ruinous effects on old masonry structures, most of them actually in use as churches, schools and institutional buildings.

It became evident that only a systematic study of the seismic vulnerability of the historical masonry structures can lead to the safeguard of a large number of old buildings and monuments which represents a relevant social, economic and cultural heritage.

\footnotetext{
* Corresponding author. Tel.: +39 3204395960; fax: +39 0916657749 .

E-mail addresses: giuseppe.giambanco@unipa.it, giuseppe.giambanco64@gmail.com (G. Giambanco).
} 
The evaluation of the seismic behavior of ancient masonry structures requires a specific procedure which synthetically consists of two phases: the first one is the investigation phase where the structural engineer collects the information about the original building and its subsequent evolution. All the data regarding the geometry of the structural elements and the physical and mechanical properties of the construction materials are also required. In the latter phase, on the base of the knowledge level reached in the previous phase, a more or less detailed mathematical model is defined and the structural analysis is performed.

A crucial point of the study is the adoption of an appropriate constitutive model for the masonry material. This choice has a strong influence on the data collecting phase and, of course, on the results of the structural analysis. Two different approaches are reported in the literature for the mechanical modeling of masonry. The macromodeling approach intentionally makes no distinction between units and joints with the aim to formulate a fictitious homogeneous and continuous material equivalent to the actual one which is discrete and composite. The alternative meso-modeling approach analyzes the masonry material as a discontinuous assembly of blocks, connected each other by mortar or dry joints at their actual position.

For the purpose of numerical modeling of heterogeneous material structures, the meso-modeling approach has been implemented in the framework of the finite element method (FEM). The mechanical behavior of mortar joints is simulated making use of zero thickness interface (ZTI) elements where the displacement discontinuities between the top and bottom sides of the element represent the primary kinematic variables.

A wide literature is devoted to the development of advanced interface constitutive laws in order to describe the damage evolution and the onset of irreversible strains and related coupled effects for different mechanical problems. These constitutive laws are expressed in terms of contact tractions and displacement discontinuities and are often formulated in a rigorous manner incorporating some concepts developed in damage mechanics, in the theory of plasticity for non standard materials and in fracture mechanics.

Interface constitutive laws based on the thermodynamics of damaging materials have been formulated by Corigliano and Allix [1] and implemented in order to simulate the mixed-mode decohesion process in composite laminates. Anisotropy of damage is taken into account in two different ways. In the first way three damage variables are assumed, two in the tangential directions and the remaining one in the normal direction. The elastic locus is expressed as a function of damage energy release rates, thermodynamic forces associated to the damage variables, and a variable damage threshold. The evolution equations of damage variables are of associative type. In the latter way the anisotropic response is obtained making use of non associative flow rules for the single damage variable.

On the same line, Alfano and Crisfield [2] proposed a damage interface model with a single damage variable whose evolution law does not fulfill a normality law. The convex elastic domain is defined as a function of the thermodynamic forces associated to fracture modes I and II. In their work particular attention is paid to the numerical implementation of the interface constitutive laws.

An interface model similar to the two above cited has been applied by William et al. [3] to the meso-modeling of thermomechanical degradation of heterogeneous materials.

Stankowski et al. [4], Lofti and Shing [5] and Lourenco and Rots [6] have proposed other interface models, formulated in a rigorous manner, which incorporate some concepts developed in the theory of plasticity for nonstandard materials and in fracture mechanics. All these models adopt Mohr-Coulomb type failure criteria and use internal variables to describe the post-peak softening behavior. Lourenco and Rots [6] adopted a cap model to take into account the joint compaction due to high compressive stresses.

A simple elastoplastic interface model has been proposed by Giambanco et al. [7]. It possesses general features similar to the previous models, but with particular attention addressed to the cohesive-frictional joint transition, taking into account the geometrical dilatancy which appears in the pure frictional stage related to the roughness of the fracture-slip surface. A tribological law is adopted to describe the evolution of contact surface and of the related variation of shear strength.

Time dependent interface laws have been developed for bonded joints in the framework of viscoplasticity for generalized standard materials by Giambanco and Fileccia Scimemi [8]. These laws are formulated in a thermodynamically consistent manner and take into account the rate dependency modifications of the fracture process zone introducing a sort of non-local instantaneous dissipation.

Recently, some authors have been concentrated in the formulation of interface laws which take into account the non linear effects of stiffness degradation, loss of adhesion and unilateral contact with related frictional phenomena. The main idea, common to the models developed by Alfano e Sacco [9], Parrinello et al. [10], Spada et al. [11] and 
Sacco e Lebon [12], is that at the micro-mechanical level the contact area can be additively decomposed in a fully damaged part and in a part with sound material. In cementitious joints this idea can be translated into the fact that during the loading path a partial decohesion occurs due to the formation and nucleation of microcracks. The measure of the projection of the damaged part on the interface plane is represented by the damage variable. The mechanical behavior of the damaged part is governed by unilateral contact and friction. The undamaged part can be considered fully elastic [9] and the onset inelastic strains can be taken into account making use of an appropriate elasto-plastic model [11].

In many cases the joint response also depends on internal stresses and strains within the contact layer. A typical example is the uniaxial compression of a masonry prism. If the specimen is assembled making use of bricks stronger and stiffer than mortar, the bed joint has the tendency to expand laterally and a confinement action is provided by bricks at the contact interfaces. The shear tractions at the interfaces result in an internal stress state which consists of triaxial compression in the mortar and bilateral tension on the horizontal plane in the bricks. This state of stress produces splitting vertical cracks in the bricks that lead to the failure of the masonry prism. On the other hand, if the mortar is stiffer than bricks material, the joint provides the confinement action on the bricks and it is subjected to a tensile stress state along the mean plane.

The ZTI concept applied to the reported example is not able to capture the illustrated response of the masonry specimen and, in terms of contact tractions, provide compression in the normal direction and zero tangential tractions.

Therefore, the usual assumption adopted in ZTI elements, that the mechanical response of the joint is governed by the contact tractions only, requires an enhancement by introducing the effects of internal stresses into the analysis.

The enhancement of the ZTI model is represented by the interphase model introduced by Giambanco and Mroz [13] and implemented by Giambanco et al. [14] in the framework of finite elements. The interphase element is a reliable contact finite element representing a thin layer joint separated by two interfaces from the adherents. The internal stresses are incorporated and the initial contact stiffness is evaluated on the base of the elastic properties of the bulk material.

The present paper deals with the nonlinear mesomodeling of masonry structures and presents the application of the interphase concept to the simulation of the mechanical behavior of head and bed mortar joints.

The principal aim of the work is to show another peculiar feature of the interphase model. Differently to ZTI model where the nonlinear phenomena in the thin layer and at the interfaces are both governed by the contact tractions and a unique constitutive law must describe the overall response, the adoption of the interphase model and the consequent distinction between contact tractions and internal stresses, allows to introduce different failure conditions for the physical interfaces and for the bulk material.

In particular, in the paper the damage of the joint material is described separately from the loss of adhesion typically occurring at the joint-adherent contact surface. Therefore, the joint stiffness degradation is related to the damage of the joint material and the attainment of a specific yield condition at the physical interfaces is responsible for the onset of irreversible displacement discontinuities.

The paper is structured as follows. In Section 2, for the sake of completeness, the interphase concept is illustrated in terms of kinematic hypotheses and the indefinite equilibrium equations are derived with the classical principle of virtual displacements. In Section 3 the elastoplastic damaging interphase constitutive equations are derived making use of a thermodynamically consistent approach. The discrete form of the constitutive equations is formulated in Section 4 for the purpose of implementation in a step by step analysis. Some details on the numerical algorithms developed and inserted in a scientific oriented finite element code are illustrated in Section 5. Finally numerical applications are reported in Section 6 in order to validate the interphase nonlinear model and to test the effectiveness of the numerical procedure.

\section{General assumptions}

Let us consider, in the Euclidean space $\Re^{3}$ referred to the orthonormal frame $\left(O, \mathbf{i}_{1}, \mathbf{i}_{2}, \mathbf{i}_{3}\right)$, a structure formed by two adherents $\Omega^{+}, \Omega^{-}$connected by a third material $\Omega^{j}$ in contact with the two bodies by means of the two physical interfaces $\Sigma^{+}$and $\Sigma^{-}$respectively, as in Fig. 1.

It is assumed that the thickness $h$ of the joint is small if compared with the characteristic dimensions of the bonded assembly. 

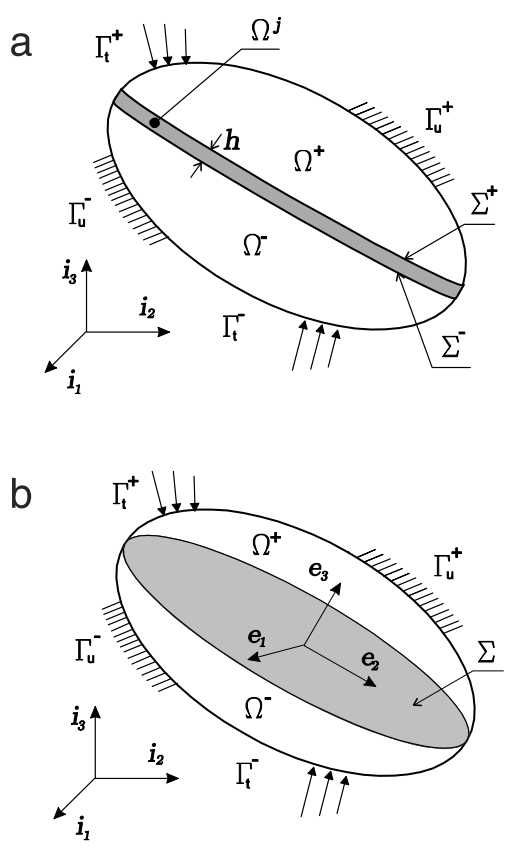

Fig. 1. Mechanical scheme of a third body interposed between two adherents.

The boundary of the two adherents is divided into two parts $\Gamma_{u}^{ \pm}$and $\Gamma_{t}^{ \pm}$, where kinematic and loading conditions are specified respectively.

The joint interacts with the two adherents through the following traction components:

$$
\mathbf{t}^{ \pm}=t_{1}^{ \pm} \mathbf{e}_{1}+t_{2}^{ \pm} \mathbf{e}_{2}+t_{3}^{ \pm} \mathbf{e}_{3}
$$

which can be considered as external surface loads for the joint.

In Eq. (1) $\mathbf{e}_{1}, \mathbf{e}_{2}$ and $\mathbf{e}_{3}$ are the unit vectors of the local reference system, with $\mathbf{e}_{3}$ oriented along the normal to the middle surface $\Sigma$ and directed towards the adherent $\Omega^{+}$.

The joint can be regarded as an interphase model. It is assumed that the fibers inside the interphase and directed along $\mathbf{e}_{3}$ are maintained rectilinear during the deformation process. In view of this hypothesis the interphase displacement field $\mathbf{u}$ can be easily obtained from the displacements $\mathbf{u}^{+}$and $\mathbf{u}^{-}$of the interfaces $\Sigma^{+}$and $\Sigma^{-}$, thus

$$
\mathbf{u}\left(x_{1}, x_{2}, x_{3}\right)=\left(\frac{1}{2}+\frac{x_{3}}{h}\right) \mathbf{u}^{+}\left(x_{1}, x_{2}\right)+\left(\frac{1}{2}-\frac{x_{3}}{h}\right) \mathbf{u}^{-}\left(x_{1}, x_{2}\right)
$$

with $x_{1}, x_{2}$ and $x_{3}$ the Cartesian coordinates in the orthonormal frame $\left(O, \mathbf{e}_{1}, \mathbf{e}_{2}, \mathbf{e}_{3}\right)$.

Since the thickness of the joint is generally small if compared to the characteristic dimensions of the adherents, we can assume the strain state $\boldsymbol{\varepsilon}$ uniform along the $\mathbf{e}_{3}$ direction and given by:

$$
\boldsymbol{\varepsilon}\left(x_{1}, x_{2}\right)=\frac{1}{h} \int_{-h / 2}^{h / 2} \nabla^{s} \mathbf{u}\left(x_{1}, x_{2}, x_{3}\right) d x_{3} .
$$

Substituting the expression (2) in (3) we have:

$$
\boldsymbol{\varepsilon}\left(x_{1}, x_{2}\right)=\frac{1}{2 h}([\mathbf{u}] \otimes \mathbf{n}+\mathbf{n} \otimes[\mathbf{u}])+\frac{1}{2} \nabla^{s}\left(\mathbf{u}^{+}+\mathbf{u}^{-}\right)
$$

where $[\mathbf{u}]=\mathbf{u}^{+}-\mathbf{u}^{-}, \mathbf{n} \equiv \mathbf{e}_{3}$ and $\nabla^{s}$ is the symmetric gradient operator defined as $\nabla^{s}=\frac{1}{2}\left(\nabla+\nabla^{T}\right)$.

Let us note that in the interphase model the joint curvatures generated by the displacement field (2) and the related flexural effect are neglected.

In order to derive the equilibrium equations of the interphase model, let us assign the virtual displacements $\delta \mathbf{u}^{+}$ and $\delta \mathbf{u}^{-}$to the interfaces $\Sigma^{+}$and $\Sigma^{-}$. The principle of virtual displacements $(P V D)$ asserts that the external work, 
produced by the contact tractions, equals the internal work developed in the thin joint. According to the hypothesis of a constant strain state along the thickness direction, the conjugate stress state is considered uniform along the same direction and the $P V D$ can be written as:

$$
\int_{\Sigma^{+}} \delta \mathbf{u}^{+} \cdot \mathbf{t}^{+} d \Sigma+\int_{\Sigma^{-}} \delta \mathbf{u}^{-} \cdot \mathbf{t}^{-} d \Sigma=h \int_{\Sigma} \delta \boldsymbol{\varepsilon}: \sigma d \Sigma
$$

Taking into account the kinematic relations (4) the virtual work equation (5) becomes

$$
\begin{aligned}
\int_{\Sigma^{+}} \delta \mathbf{u}^{+} \cdot \mathbf{t}^{+} d \Sigma+\int_{\Sigma^{-}} \delta \mathbf{u}^{-} \cdot \mathbf{t}^{-} d \Sigma= & \frac{1}{2} \int_{\Sigma}(\delta[\mathbf{u}] \otimes \mathbf{n}+\mathbf{n} \otimes \delta[\mathbf{u}]): \sigma d \Sigma \\
& +\frac{h}{2} \int_{\Sigma}\left[\nabla^{s}\left(\delta \mathbf{u}^{+}+\delta \mathbf{u}^{-}\right)\right]: \boldsymbol{\sigma} d \Sigma .
\end{aligned}
$$

Let us apply the divergence theorem to the second term of the right-hand side in Eq. (6):

$$
\int_{\Sigma}\left[\nabla^{s}\left(\delta \mathbf{u}^{+}+\delta \mathbf{u}^{-}\right)\right]: \sigma d \Sigma=\int_{\Gamma} \mathbf{m} \cdot \boldsymbol{\sigma} \cdot\left(\delta \mathbf{u}^{+}+\delta \mathbf{u}^{-}\right) d \Gamma+-\int_{\Sigma}\left(\delta \mathbf{u}^{+}+\delta \mathbf{u}^{-}\right) \cdot \operatorname{div} \boldsymbol{\sigma} d \Sigma,
$$

where $\Gamma$ represents the perimeter of the joint middle plane $\Sigma$ and $\mathbf{m}$ is the normal to the perimeter specifying lateral surface tractions. Substituting Eq. (7) in the equality (6) and assuming that $\Sigma=\Sigma^{+}=\Sigma^{-}$, we derive the $P V D$ particularized for the interphase model, namely:

$$
\begin{aligned}
& \int_{\Sigma} \delta \mathbf{u}^{+} \cdot\left(\mathbf{t}^{+}-\boldsymbol{\sigma} \cdot \mathbf{n}+\frac{h}{2} \operatorname{div} \boldsymbol{\sigma}\right) d \Sigma+\int_{\Sigma} \delta \mathbf{u}^{-} \cdot\left(\mathbf{t}^{-}+\boldsymbol{\sigma} \cdot \mathbf{n}+\frac{h}{2} \operatorname{div} \boldsymbol{\sigma}\right) d \Sigma \\
& \quad=\frac{h}{2} \int_{\Gamma} \mathbf{m} \cdot \boldsymbol{\sigma} \cdot\left(\delta \mathbf{u}^{+}+\delta \mathbf{u}^{-}\right) d \Gamma .
\end{aligned}
$$

Since the previous equality holds for all virtual displacement fields, it generates the local equilibrium relations of the interphase model:

$$
\begin{aligned}
& \mathbf{t}^{+}-\boldsymbol{\sigma} \cdot \mathbf{n}+\frac{h}{2} \operatorname{div} \boldsymbol{\sigma}=0, \quad \mathbf{t}^{-}+\boldsymbol{\sigma} \cdot \mathbf{n}+\frac{h}{2} \operatorname{div} \boldsymbol{\sigma}=0 \quad \text { on } \Sigma \\
& \mathbf{m} \cdot \boldsymbol{\sigma}=0 \text { on } \Gamma .
\end{aligned}
$$

The indefinite equations (9), making the positions $\overline{\mathbf{t}}=\frac{1}{2}\left(\mathbf{t}^{+}+\mathbf{t}^{-}\right)$and $\tilde{\mathbf{t}}=\frac{1}{2}\left(\mathbf{t}^{+}-\mathbf{t}^{-}\right)$can be rewritten in the following form:

$$
\begin{aligned}
& \overline{\mathbf{t}}+\frac{h}{2} \operatorname{div} \boldsymbol{\sigma}=0 \quad \text { on } \Sigma \\
& \widetilde{\mathbf{t}}=\boldsymbol{\sigma} \cdot \mathbf{n} \text { on } \Sigma .
\end{aligned}
$$

Eqs. (11) and (12) can be regarded as internal and external equilibrium equations of the interphase and Eq. (10) represents the equilibrium boundary conditions.

\section{Interphase constitutive model}

In the present section the thermodynamically consistent constitutive laws for the interphase are developed taking into account the stiffness degradation and the onset of inelastic strains which occur along the loading process if certain limit conditions are violated.

The basic kinematical hypothesis is the additive decomposition of the total strain in the internal $(i)$ and contact $(c)$ parts and, for the contact strain only, a further decomposition in the elastic $(e)$ and inelastic $(p)$ parts:

$$
\begin{aligned}
& \boldsymbol{\varepsilon}=\boldsymbol{\varepsilon}^{i}+\boldsymbol{\varepsilon}^{c} \\
& \boldsymbol{\varepsilon}^{c}=\boldsymbol{\varepsilon}^{c e}+\boldsymbol{\varepsilon}^{c p}
\end{aligned}
$$


with

$$
\boldsymbol{\varepsilon}^{i}=\mathbf{A} \boldsymbol{\varepsilon} \mathbf{A}
$$

being $\mathbf{A}=\mathbf{I}-\mathbf{n} \otimes \mathbf{n}$ and $\mathbf{I}$ the unit second order tensor.

In order to comply with thermodynamic requirements, the interphase Helmholtz free energy is introduced in the following form:

$$
\Psi\left(\varepsilon^{i}, \varepsilon^{c}, \varepsilon^{c p}, \omega\right)=\Psi^{i}\left(\varepsilon^{i}, \omega\right)+\Psi^{c}\left(\varepsilon^{c}, \varepsilon^{c p}\right)+\Psi^{i c}\left(\varepsilon^{i}, \varepsilon^{c}, \varepsilon^{c p}, \omega\right) ;
$$

where $\Psi^{i}$ and $\Psi^{c}$ represent the free energies related to the internal and contact parts of the strain state respectively and $\Psi^{i c}$ is the mixed term of the free energy which takes into account the co-presence of the contact and internal strains.

The hypothesis assumed to develop the constitutive laws is that damage occurs in the bulk material, therefore the damage tensor $\omega$ appears in the two terms of the total free energy which depend on the internal strains. In this way the constitutive model takes into account the onset of microvoids and fractures along the thickness of the joint. On the other hand, debonding of the joint from the adherents, sliding and fractures developing on surfaces parallel to the middle plane of the interphase, are modeled using elastoplasticity. The inelastic contact strains $\varepsilon^{c p}$ represent the internal variables.

On the base of the previous considerations a single scalar damage variable $\omega$ governs the loss of stiffness of the bulk material. The adopted expression of the free energy part related to the internal strains only is:

$$
\Psi^{i}=\Psi^{i e}+\Psi^{i d},
$$

where $\Psi^{i e}$ and $\Psi^{i d}$ are the elastic and intrinsic parts of the internal free energy, having the following explicit expressions

$$
\begin{aligned}
\Psi^{i e} & =\frac{1}{2}\left[\lambda\left\langle\operatorname{tr} \varepsilon^{i}\right\rangle^{2}+2 \mu \varepsilon^{i}: \varepsilon^{i}\right](1-\omega)+\frac{1}{2} \lambda\left\langle-\operatorname{tr} \varepsilon^{i}\right\rangle^{2}, \\
\Psi^{i d} & =-h_{d}\left[\xi_{d}+\ln \left(1-\xi_{d}\right)\right]
\end{aligned}
$$

with $\langle\cdot\rangle$ representing the McAulay brackets.

The scalar damage variable appearing in Eq. (18) varies in the range $0 \leq \omega \leq 1$, with the inferior and superior limits having the meaning of pristine and fully damaged bulk material, respectively. The $\Psi^{i d}$ term is the potential which governs the softening response associated to the damage onset and growth. It depends on the internal variable $\xi_{d}$ and on the material parameter $h_{d}$.

Debonding and sliding deformation mechanisms at the physical interfaces are described making use of the following additive decomposition of the contact free energy in the elastic (ce) and intrinsic ( $c p$ ) parts

$$
\Psi^{c}=\Psi^{c e}+\Psi^{c p},
$$

where the explicit expressions of the two parts are:

$$
\begin{aligned}
\Psi^{c e} & =\frac{1}{2}\left[\lambda \operatorname{tr}^{2}\left(\varepsilon^{c}-\varepsilon^{c p}\right)+2 \mu\left(\varepsilon^{c}-\varepsilon^{c p}\right):\left(\varepsilon^{c}-\varepsilon^{c p}\right)\right], \\
\Psi^{c p} & =\frac{1}{2} h_{p} \xi_{p}^{2},
\end{aligned}
$$

being $\xi_{p}$ the internal elastoplastic variable and $h_{p}$ a material parameter specifying isotropic hardening/softening interface response.

Finally the explicit expression of the mixed term of the free energy is:

$$
\Psi^{i c}=\lambda\left[(1-\omega)\left\langle\operatorname{tr} \varepsilon^{i}\right\rangle-\left\langle-\operatorname{tr} \varepsilon^{i}\right\rangle\right] \operatorname{tr}\left(\varepsilon^{c}-\varepsilon^{c p}\right) .
$$




\subsection{State equations}

In order to derive the interphase constitutive equations, the second principle of thermodynamics, including also the balance equation (first principle) can be applied in the form of the Clausius-Duhem inequality. This inequality for an isothermal purely mechanical evolutive process reads as

$$
D=\sigma: \dot{\varepsilon}-\dot{\Psi} \geq 0,
$$

where $D$ is the interphase dissipation for unit surface or net entropy production.

The general rate form of the Helmholtz free energy (16) has the following expression:

$$
\begin{aligned}
\dot{\Psi}= & \left(\frac{\partial \Psi^{i e}}{\partial \boldsymbol{\varepsilon}^{i}}+\frac{\partial \Psi^{i c}}{\partial \boldsymbol{\varepsilon}^{i}}\right): \dot{\boldsymbol{\varepsilon}}^{i}+\left(\frac{\partial \Psi^{c e}}{\partial \boldsymbol{\varepsilon}^{\boldsymbol{c}}}+\frac{\partial \Psi^{i c}}{\partial \boldsymbol{\varepsilon}^{\boldsymbol{c}}}\right): \dot{\boldsymbol{\varepsilon}}^{c}+\left(\frac{\partial \Psi^{c e}}{\partial \boldsymbol{\varepsilon}^{\boldsymbol{c p}}}+\frac{\partial \Psi^{i c}}{\partial \boldsymbol{\varepsilon}^{c \boldsymbol{p}}}\right): \dot{\boldsymbol{\varepsilon}}^{c p} \\
& +\left(\frac{\partial \Psi^{i e}}{\partial \omega}+\frac{\partial \Psi^{i c}}{\partial \omega}\right) \dot{\omega}+\frac{\partial \Psi^{i d}}{\partial \xi^{d}} \dot{\xi}^{d}+\frac{\partial \Psi^{c p}}{\partial \xi^{p}} \dot{\xi}^{p} .
\end{aligned}
$$

Particularizing Eq. (24) for a purely elastic incremental deformation process, $\left(\dot{\boldsymbol{\varepsilon}}^{c p}=\mathbf{0}, \dot{\omega}=\dot{\xi}^{d}=\dot{\xi}^{p}=0\right)$, assuming a decomposition of the stress state similar to that used for the strain state

$$
\boldsymbol{\sigma}=\boldsymbol{\sigma}^{i}+\boldsymbol{\sigma}^{c}, \quad \text { being } \boldsymbol{\sigma}^{i}=\mathbf{A} \boldsymbol{\sigma} \mathbf{A}
$$

and considering the adopted expressions of the free energy parts (16)-(23), the elastic stress-strain equations can be derived, thus

$$
\begin{aligned}
\boldsymbol{\sigma}^{i}= & \frac{\partial \Psi^{i e}}{\partial \boldsymbol{\varepsilon}^{i}}+\frac{\partial \Psi^{i c}}{\partial \boldsymbol{\varepsilon}^{i}}=\lambda\left\{(1-\omega)\left[\left\langle\operatorname{tr} \boldsymbol{\varepsilon}^{i}\right\rangle+H\left(\operatorname{tr} \boldsymbol{\varepsilon}^{i}\right) \operatorname{tr}\left(\boldsymbol{\varepsilon}^{c}-\boldsymbol{\varepsilon}^{c p}\right)\right]+\right. \\
& \left.-\left\langle-\operatorname{tr} \boldsymbol{\varepsilon}^{i}\right\rangle+H\left(-\operatorname{tr} \boldsymbol{\varepsilon}^{i}\right) \operatorname{tr}\left(\boldsymbol{\varepsilon}^{c}-\boldsymbol{\varepsilon}^{c p}\right)\right\} \mathbf{A}+2 \mu(1-\omega) \boldsymbol{\varepsilon}^{i}, \\
\boldsymbol{\sigma}^{c}= & \frac{\partial \Psi^{c e}}{\partial \boldsymbol{\varepsilon}^{\boldsymbol{c}}}+\frac{\partial \Psi^{i c}}{\partial \boldsymbol{\varepsilon}^{c}}=\lambda\left[(1-\omega)\left\langle\operatorname{tr} \boldsymbol{\varepsilon}^{i}\right\rangle-\left\langle-\operatorname{tr} \boldsymbol{\varepsilon}^{i}\right\rangle+\operatorname{tr}\left(\boldsymbol{\varepsilon}^{c}-\boldsymbol{\varepsilon}^{c p}\right)\right](\boldsymbol{I}-\boldsymbol{A})+2 \mu\left(\boldsymbol{\varepsilon}^{c}-\boldsymbol{\varepsilon}^{c p}\right),
\end{aligned}
$$

with $H(\cdot)$ the unit step function. The interphase state equations are completed by the definition of the static variables $\chi^{p}$ and $\chi^{d}$ conjugate to the internal variables $\xi^{p}$ and $\xi^{d}$ respectively, and of the thermodynamic force $\zeta$ conjugate to the damage variable $\omega$ :

$$
\begin{aligned}
\chi^{p} & :=\frac{\partial \Psi^{c p}}{\partial \xi^{p}}=h_{p} \xi^{p} \\
\chi^{d} & :=\frac{\partial \Psi^{i d}}{\partial \xi^{d}}=h_{d} \frac{\xi^{d}}{1-\xi^{d}} \\
\zeta & :=-\left(\frac{\partial \Psi^{i e}}{\partial \omega}+\frac{\partial \Psi^{i c}}{\partial \omega}\right)=\frac{1}{2}\left[\lambda\left\langle\operatorname{tr} \boldsymbol{\varepsilon}^{i}\right\rangle^{2}+2 \mu \boldsymbol{\varepsilon}^{i}: \boldsymbol{\varepsilon}^{i}\right]+\lambda\left\langle\operatorname{tr} \boldsymbol{\varepsilon}^{i}\right\rangle \operatorname{tr}\left(\boldsymbol{\varepsilon}^{c}-\boldsymbol{\varepsilon}^{c p}\right) .
\end{aligned}
$$

Making use of the elastic strain-stress equation and of the previous positions, the final expression of the instantaneous dissipation is obtained:

$$
D=\sigma^{c}: \dot{\boldsymbol{\varepsilon}}^{c p}-\chi^{p} \dot{\xi}^{p}+\zeta \dot{\omega}-\chi^{d} \dot{\xi}^{d} \geq 0 .
$$

It can be noticed that the proposed decomposition of the total strain in the internal and contact parts together with the assumed form of the intrinsic free energies, from a theoretical point of view, leads us to state that the interphase mechanisms inducing micro structural changes can be separately activated. In fact, the instantaneous dissipation appears split in two parts, namely:

$$
D=D^{c}+D^{i}
$$


with

$$
\begin{aligned}
& D^{c}=\sigma^{c}: \dot{\boldsymbol{\varepsilon}}^{c p}-\chi^{p} \dot{\xi}^{p} \geq 0 \\
& D^{i}=\zeta \dot{\omega}-\chi^{d} \dot{\xi}^{d} \geq 0
\end{aligned}
$$

where $D^{c}$ is the dissipation related to the opening and sliding phenomena at the physical interfaces and $D^{i}$ is the dissipation associated to the damage in the bulk material.

\subsection{Yield conditions and flow rules}

In order to regulate the activation of each dissipative mechanism, two different yield functions are defined in the space of the proper static variables, namely:

$$
\Phi^{p}\left(\sigma^{c}, \chi^{p}\right) \leq 0, \quad \Phi^{d}\left(\zeta, \chi^{d}\right) \leq 0
$$

where $\Phi^{p}$ is the classical plastic yield function specifying the elastic contact domain and $\Phi^{d}$ is the damage activation function, both assumed convex.

The activation of each dissipation mechanism can be effectively described by a variational formulation which is represented by the generalized principle of maximum intrinsic dissipation:

$$
\max _{\sigma^{c}, \chi^{p}, \zeta, \chi^{d}}\left(\boldsymbol{\sigma}^{c}: \dot{\boldsymbol{\varepsilon}}^{c p}-\chi^{p} \dot{\xi}^{p}+\zeta \dot{\omega}-\chi^{d} \dot{\xi}^{d}\right)
$$

subject to the constraints (36). The Kuhn-Tucker conditions of the maximum constrained problem provide the plastic and damage evolution laws of the interphase:

$$
\begin{aligned}
& \dot{\boldsymbol{\varepsilon}}^{c p}=\dot{\Lambda}^{p} \frac{\partial \Phi^{p}}{\partial \sigma^{c}}, \quad \dot{\xi}^{p}=-\dot{\Lambda}^{p} \frac{\partial \Phi^{p}}{\partial \chi^{p}}, \\
& \dot{\omega}=\dot{\Lambda}^{d} \frac{\partial \Phi^{d}}{\partial \zeta}, \quad \dot{\xi}^{d}=-\dot{\Lambda}^{d} \frac{\partial \Phi^{d}}{\partial \chi^{d}}, \\
& \Phi^{p}\left(\sigma^{c}, \chi^{p}\right) \leq 0, \quad \Phi^{d}\left(\zeta, \chi^{d}\right) \leq 0, \\
& \dot{\Lambda}^{p} \geq 0, \quad \dot{\Lambda}^{d} \geq 0, \quad \dot{\Lambda}^{d} \Phi^{d}\left(\zeta, \chi^{d}\right)=0,
\end{aligned}
$$

being $\dot{\Lambda}^{p}$ and $\dot{\Lambda}^{d}$ the plastic and damage multipliers, respectively. In the present study the elasto-plastic convex domain is defined by the intersection of the classical Mohr-Coulomb bilinear function with a tension cut-off:

$$
\begin{aligned}
& \Phi^{p 1}\left(\boldsymbol{\sigma}^{c}, \chi^{p}\right)=\left|\boldsymbol{\tau}^{c}\right|+\sigma^{c} \tan \phi-c_{0}\left(1-\chi^{p}\right), \\
& \Phi^{p 2}\left(\boldsymbol{\sigma}^{c}, \chi^{p}\right)=\sigma^{c}-\sigma_{0}\left(1-\chi^{p}\right),
\end{aligned}
$$

where $\boldsymbol{\tau}^{c}$ and $\sigma^{c}$ are the tangential stress vector and the normal stress component of the contact stresses, $\phi$ is the friction angle, $c_{0}$ and $\sigma_{0}$ the cohesion and tensile strength of the virgin interfaces.

The two yield functions are depicted in Fig. 2. The following four zones can be distinguished:

I elastic zone, $\Phi^{p 1}<0 \Phi^{p^{2}}<0$;

II plastic activation in shear, $\Phi^{p 1}=0 \Phi^{p 2}<0$;

III plastic activation in tension, $\Phi^{p 1}<0 \Phi^{p^{2}}=0$;

IV plastic activation in tension and shear (corner), $\Phi^{p 1}=0 \Phi^{p 2}=0$.

The damage activation function is linear and the first activation occurs when the thermodynamic force reaches the relative threshold value $\zeta_{0}$ :

$$
\Phi^{d}\left(\zeta, \chi^{d}\right)=\zeta-\zeta_{0}-\chi^{d}
$$




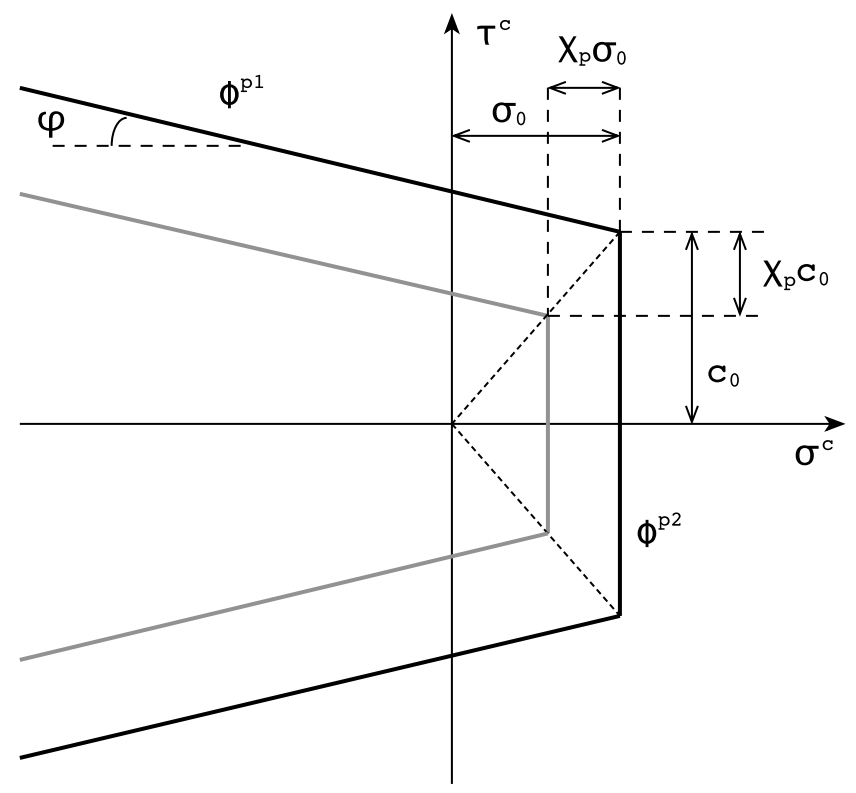

Fig. 2. Bilinear plastic limit condition represented in the plane stress space.

\subsection{Joint dilatancy}

Dilatancy is that property of a material by which it increases in volume when it is sheared. For this reason the effect is sometime called shear thickening.

In masonry material dilatancy occurs in the bed joints when they are subjected to compression and shear loads and, if the normal displacements are bounded, the overall effect is an increase of the normal load resulting in an increased shear strength. Dilatancy has been observed experimentally by Atkinson et al. [15] who tested several masonry specimens under constant normal load and cyclic shear displacement. When the peak nominal shear stress is reached, the normal displacement increases with the shear displacement. During the unloading phase the joint contraction is observed until the reverse sliding takes place with a new expansion of the joint. After some cycles dilatancy is less pronounced. The mechanical interpretation of this phenomenon is that during the decohesion process micro-voids and fractures appear making the sliding plane rough. Therefore, expansion and contraction of the joint is the kinematical effect related to the sliding along asperities $[7,10]$.

In the present work dilatancy is considered adopting for the irreversible contact strains a non-associated flow rule related to the sliding limit condition.

Eqs. (38) are then replaced using the well-known generalized Koiter's form [16]:

$$
\dot{\boldsymbol{\varepsilon}}^{c p}=\dot{\Lambda}^{p 1} \frac{\partial G^{p}}{\partial \boldsymbol{\sigma}^{c}}+\dot{\Lambda}^{p 2} \frac{\partial \Phi^{p 2}}{\partial \boldsymbol{\sigma}^{c}}, \quad \dot{\xi}^{p}=-\dot{\Lambda}^{p 1} \frac{\partial \Phi^{p 1}}{\partial \chi^{p}}-\dot{\Lambda}^{p 2} \frac{\partial \Phi^{p 2}}{\partial \chi^{p}},
$$

where $\dot{\Lambda}^{p 1}, \dot{\Lambda}^{p 2}$ are the plastic multipliers which satisfy the following complementarity relations:

$$
\Phi^{p 1} \leq 0, \quad \Phi^{p 2} \leq 0, \quad \dot{\Lambda}^{p 1} \geq 0, \quad \dot{\Lambda}^{p 2} \geq 0, \quad \Phi^{p 1} \dot{\Lambda}^{p 1}+\Phi^{p^{2}} \dot{\Lambda}^{p 2}=0,
$$

which describe all the loading/unloading conditions. The plastic potential related to the limit condition (43) is expressed by the following function:

$$
G^{p}\left(\sigma^{c}, \chi^{p}\right)=\left|\boldsymbol{\tau}^{c}\right|+\sigma^{c}\left(1-\chi^{p}\right) \tan \delta,
$$

with $\delta \in[0, \phi]$ dilatancy angle. From the above reported expression it is clear that $\delta$ is the initial dilatancy angle and it decreases along the loss of cohesion process according to the experimental observations. 


\section{Discrete interphase constitutive laws}

In order to implement the non linear interphase model in a structural numerical code, the constitutive equations have to be integrated for a given incremental strain history with assigned initial conditions. Let $[0, T] \subset \Re$ be the time interval of interest and assume that the state variables at time $t_{n} \in[0, T]$ are known, that is

$$
\left\{\boldsymbol{\varepsilon}_{n}, \boldsymbol{\varepsilon}_{n}^{c p}, \omega_{n}, \xi_{n}^{p}, \xi_{n}^{d}\right\} \quad \text { given at assigned } t_{n} .
$$

We shall denote $\Delta \boldsymbol{\varepsilon}$ the assigned increment of total strain produced in the time step $\left(t_{n+1}-t_{n}\right)$ with $t_{n+1} \in[0, T]$. The central problem of the integration procedure is to update the field variables (49) to $t_{n+1}$ in a manner consistent with the interphase constitutive equations reported in the previous section.

Eqs. (38) and (39) define a non linear evolution problem with initial conditions (49) subjected to the unilateral constraints (40)-(42). This continuum problem can be transformed into a discrete one by applying the implicit backward-Euler difference scheme:

$$
\begin{aligned}
& \boldsymbol{\varepsilon}_{n+1}=\boldsymbol{\varepsilon}_{n}+\Delta \boldsymbol{\varepsilon}, \\
& \boldsymbol{\varepsilon}_{n+1}^{c p}=\boldsymbol{\varepsilon}_{n}^{c p}+\left.\Delta \Lambda^{p 1} \frac{\partial G^{p}}{\partial \boldsymbol{\sigma}^{c}}\right|_{n+1}+\left.\Delta \Lambda^{p 2} \frac{\partial \Phi^{p 2}}{\partial \boldsymbol{\sigma}^{c}}\right|_{n+1}, \\
& \Delta \xi^{p}=-\left.\Delta \Lambda^{p 1} \frac{\partial \Phi^{p 1}}{\partial \chi^{p}}\right|_{n+1}-\left.\Delta \Lambda^{p 2} \frac{\partial \Phi^{p 2}}{\partial \chi^{p}}\right|_{n+1}, \\
& \Delta \omega=\left.\Delta \Lambda^{d} \frac{\partial \Phi^{d}}{\partial \zeta}\right|_{n+1}, \\
& \Delta \xi^{d}=-\left.\Delta \Lambda^{d} \frac{\partial \Phi^{d}}{\partial \chi^{d}}\right|_{n+1} .
\end{aligned}
$$

The discrete counterpart of loading/unloading conditions along the time step can be written as

$$
\begin{aligned}
& \Phi_{n+1}^{p 1}=0, \quad \Phi_{n+1}^{p 2}=0, \quad \Delta \Lambda^{p 1} \geq 0, \quad \Delta \Lambda^{p 2} \geq 0, \quad \Phi_{n+1}^{p 1} \Delta \Lambda^{p 1}+\Phi_{n+1}^{p 2} \Delta \Lambda^{p 2}=0 ; \\
& \Phi_{n+1}^{d}=0, \quad \Delta \Lambda^{d} \geq 0, \quad \Phi_{n+1}^{d} \Delta \Lambda^{d}=0 .
\end{aligned}
$$

The above discrete equations may be considered in the framework of a two-step algorithm which splits the previous problem in an additive manner, in an elastic trial predictor stage and in a plastic-damage corrector stage. In the time step $\left(t_{n+1}-t_{n}\right)$ the elastic predictor stage leads to the following results:

$$
\text { predictor } \rightarrow \begin{cases}\boldsymbol{\varepsilon}_{n+1}^{c p^{\text {trial }}}=\boldsymbol{\varepsilon}_{n}^{c p}, & \omega_{n+1}^{\text {trial }}=\omega_{n}, \\ \xi_{n+1}^{p^{\text {trial }}}=\xi_{n}^{p}, & \xi_{n+1}^{d^{\text {trial }}}=\xi_{n}^{d} .\end{cases}
$$

The dependent variables assume the following values:

$$
\begin{aligned}
\boldsymbol{\sigma}_{n+1}^{t^{\text {trial }}=} & \lambda\left\{\left(1-\omega_{n}\right)\left[\left\langle\operatorname{tr} \boldsymbol{\varepsilon}_{n+1}^{i}\right\rangle+H\left(\operatorname{tr} \boldsymbol{\varepsilon}_{n+1}^{i}\right) \operatorname{tr}\left(\boldsymbol{\varepsilon}_{n+1}^{c}-\boldsymbol{\varepsilon}_{n}^{c p}\right)\right]-\left\langle-\operatorname{tr} \boldsymbol{\varepsilon}_{n+1}^{i}\right\rangle\right. \\
& \left.+H\left(-\operatorname{tr} \boldsymbol{\varepsilon}_{n+1}^{i}\right) \operatorname{tr}\left(\boldsymbol{\varepsilon}_{n+1}^{c}-\boldsymbol{\varepsilon}_{n}^{c p}\right)\right\} \mathbf{A}+2 \mu\left(1-\omega_{n}\right) \boldsymbol{\varepsilon}_{n+1}^{i}, \\
\boldsymbol{\sigma}_{n+1}^{c^{\text {trial }}}= & \lambda\left[\left(1-\omega_{n}\right)\left\langle\operatorname{tr} \boldsymbol{\varepsilon}_{n+1}^{i}\right\rangle-\left\langle-\operatorname{tr} \boldsymbol{\varepsilon}_{n+1}^{i}\right\rangle+\operatorname{tr}\left(\boldsymbol{\varepsilon}_{n+1}^{c}-\boldsymbol{\varepsilon}_{n}^{c p}\right)\right](\boldsymbol{I}-\boldsymbol{A})+2 \mu\left(\boldsymbol{\varepsilon}_{n+1}^{c}-\boldsymbol{\varepsilon}_{n}^{c p}\right), \\
\chi_{n+1}^{p^{t r i a l}}= & h_{p} \xi_{n}^{p}, \\
\chi_{n+1}^{d^{t r i a l}}= & h_{d} \frac{\xi_{n}^{d}}{1-\xi_{n}^{d}}, \\
\zeta^{\text {trial }}= & \frac{1}{2}\left[\lambda\left\langle\operatorname{tr} \boldsymbol{\varepsilon}_{n+1}^{i}\right\rangle^{2}+2 \mu \boldsymbol{\varepsilon}_{n+1}^{i}: \boldsymbol{\varepsilon}_{n+1}^{i}\right]+\lambda\left\langle\operatorname{tr} \boldsymbol{\varepsilon}_{n+1}^{i}\right\rangle \operatorname{tr}\left(\boldsymbol{\varepsilon}_{n+1}^{c}-\boldsymbol{\varepsilon}_{n}^{c p}\right) .
\end{aligned}
$$


The process is elastic and the correction procedure is not activated if the following inequalities are satisfied:

$$
\begin{aligned}
& \Phi_{n+1}^{p 1^{\text {trial }}}\left(\sigma_{n+1}^{c^{\text {trial }}}, \chi_{n+1}^{p^{\text {trial }}}\right)<0, \quad \Phi_{n+1}^{p 2^{\text {trial }}}\left(\sigma_{n+1}^{c^{\text {trial }}}, \chi_{n+1}^{p^{\text {trial }}}\right)<0, \\
& \Phi_{n+1}^{d^{\text {trial }}}\left(\zeta_{n+1}^{\text {trial }}, \chi_{n+1}^{d^{\text {trial }}}\right)<0 .
\end{aligned}
$$

Otherwise the trial quantities are not feasible and the corrector stage is performed. In order to illustrate the corrector stage let us consider the most complex case where none of the inequalities (63) is satisfied. We assume [17] that trial values of the yield functions and of the damage activation function rule the loading/unloading conditions. Hence plastic multipliers $\Delta \Lambda^{p 1}, \Delta \Lambda^{p 2}$ and damage multiplier $\Delta \Lambda^{d}$ are assumed positive. Complementarity conditions (55), (56) give:

$$
\begin{aligned}
& \Phi_{n+1}^{p 1}\left(\sigma_{n+1}^{c}, \chi_{n+1}^{p}\right)=\left|\boldsymbol{\tau}_{n+1}^{c}\right|+\sigma_{n+1}^{c} \tan \phi-c_{0}\left(1-\chi_{n+1}^{p}\right)=0, \\
& \Phi_{n+1}^{p 2}\left(\sigma_{n+1}^{c}, \chi_{n+1}^{p}\right)=\sigma_{n+1}^{c}-\sigma_{0}\left(1-\chi_{n+1}^{p}\right)=0, \\
& \Phi_{n+1}^{d}\left(\zeta_{n+1}, \chi_{n+1}^{d}\right)=\zeta_{n+1}-\zeta_{0}-\chi_{n+1}^{d}=0 .
\end{aligned}
$$

Making use of Eqs. (49)-(54) and (58)-(62), and (64)-(66) at the end of the corrector stage are written as

$$
\begin{aligned}
\Phi_{n+1}^{p 1}\left(\Delta \Lambda^{p 1}, \Delta \Lambda^{p 2}, \Delta \Lambda^{d}\right)= & \Phi_{n+1}^{p 1^{\text {trial }}}-\left|\mu \frac{\boldsymbol{\tau}_{n+1}^{c^{\text {trial }}}}{\left|\boldsymbol{\tau}_{n+1}^{c^{\text {trial }}}\right|} \Delta \Lambda^{p 1}\right|+ \\
& -\left[(\lambda+2 \mu) \beta+\lambda \Delta \Lambda^{d}\left\langle\operatorname{tr} \boldsymbol{\varepsilon}_{n+1}^{i}\right\rangle\right] \tan \phi+c_{0} \Delta \chi^{p}=0, \\
\Phi_{n+1}^{p 2}\left(\Delta \Lambda^{p 1}, \Delta \Lambda^{p 2}, \Delta \Lambda^{d}\right)= & \Phi_{n+1}^{p 2^{\text {trial }}}-(\lambda+2 \mu) \beta+-\lambda \Delta \Lambda^{d}\left\langle\operatorname{tr} \boldsymbol{\varepsilon}_{n+1}^{i}\right\rangle+\sigma_{0} \Delta \chi^{p}=0, \\
\Phi_{n+1}^{d}\left(\Delta \Lambda^{p 1}, \Delta \Lambda^{p 2}, \Delta \Lambda^{d}\right)= & \Phi_{n+1}^{d^{\text {trial }}}-\lambda \operatorname{tr} \varepsilon_{n+1}^{i} \beta-\Delta \chi^{d}=0,
\end{aligned}
$$

where

$$
\begin{aligned}
& \beta=\left(1-\chi_{n}^{p}\right) \tan \phi \Delta \Lambda^{p 1}+c_{0} h_{p} \tan \phi\left(\Delta \Lambda^{p 1}\right)^{2}+\sigma_{0} h_{p} \tan \phi \Delta \Lambda^{p 1} \Delta \Lambda^{p 2}+\Delta \Lambda^{p 2} \\
& \Delta \chi^{p}=-h_{p}\left(c_{0} \Delta \Lambda^{p 1}+\sigma_{0} \Delta \Lambda^{p 2}\right) ; \quad \Delta \chi^{d}=h_{d} \frac{\Delta \Lambda^{d}}{\left(1-\xi_{n}^{d}\right)\left(1-\xi_{n}^{d}-\Delta \Lambda^{d}\right)} .
\end{aligned}
$$

Eqs. (67)-(69) represent a system of three non linear equations in the three unknowns $\Delta \Lambda^{p 1}, \Delta \Lambda^{p 2}$ and $\Delta \Lambda^{d}$ which is solved by a local Newton-Raphson numerical procedure. The same strategy is applied for other cases of yield surfaces activation. After the corrector stage the initial hypotheses are checked and if are not confirmed the procedure is restarted with the new set of active surfaces.

\section{Fem analysis and numerical algorithms}

In order to test the effectiveness of the proposed non linear interphase laws for the solution of boundary value problems regarding masonry structures, made up of blocks connected each other by mortar joints, the finite element method has been chosen to obtain the discretized version of the equilibrium equations. Space discretization has been achieved employing two-dimensional 4-nodes isoparametric elements simulating the blocks and 4-nodes interphase elements for the mortar joints.

In two-dimensional models of masonry structures the plane stress or plain strain assumption is adopted depending on the geometry of the structural element. A specific study on the range of validity of the two different assumptions has been developed by Anthoine [18]. The principal conclusion is that in the linear range both provide satisfactory results while in the non linear range the plane stress assumption may lead to inaccurate results.

In this case the plane stress assumption is adopted because the implementation in the finite element code is finalized to the study of masonry walls where the thickness is quite small if compared to the other dimensions. In the following examples the plane stress condition is also used to develop numerical simulations on masonry specimens under the 
assumption that the non linearities are concentrated at the joint level and the block material is indefinitely elastic. In view of this last assumption inaccurate results related to the plane stress condition can be considered of the same order of the numerical approximations.

The interphase element implementation has been developed according to the procedure presented in [14] and it has been incorporated in a widely used scientific oriented finite element code (FEAP). In the cited paper the numerical performance of the interphase element is investigated in detail. It has been observed that, if the conventional Gauss quadrature is adopted for the numerical integrations and the interphase stiffness is higher than that one of the adherents, the element shows an unacceptable oscillatory profile of the tangential tractions. The numerical defect, justified by the shear locking of the element, can be avoided using classical numerical strategies as the Enhanced Assumed Strain (EAS) and Selective Reduced Integration (SRI).

In the present work the SRI strategy has been preferred because it does not need the introduction of additional variables and the modification of the stiffness matrix, important requirement for non-linear applications. Therefore, after an appropriate additive decomposition of the stiffness matrix, two different integration formulae have been used: one with a single Gauss point for the part of the stiffness matrix related to the shear energy and one with two Gauss points for the remaining part.

The non linear discrete interphase laws have then been implemented at the sampling point of the finite element where in the time step $\left(t_{n+1}-t_{n}\right)$ the increment of the total strain $\Delta \boldsymbol{\varepsilon}_{n+1}$ is provided.

In the elastic predictor stage the trial values of internal and contact stresses, of the thermodynamic force and of the other static variables are evaluated making use of Eqs. (58)-(62). The damage and plastic limit conditions are checked and if any is violated the corrector stage is performed. In the case of multi-limit conditions the violation of a constraint does not insure that the constraint is active, therefore in the corrector stage the trial set of active constraints is checked after the correction. The elastic predictor stage and corrector stage pseudo-codes are illustrated in Algorithms 1 and 2 , respectively.

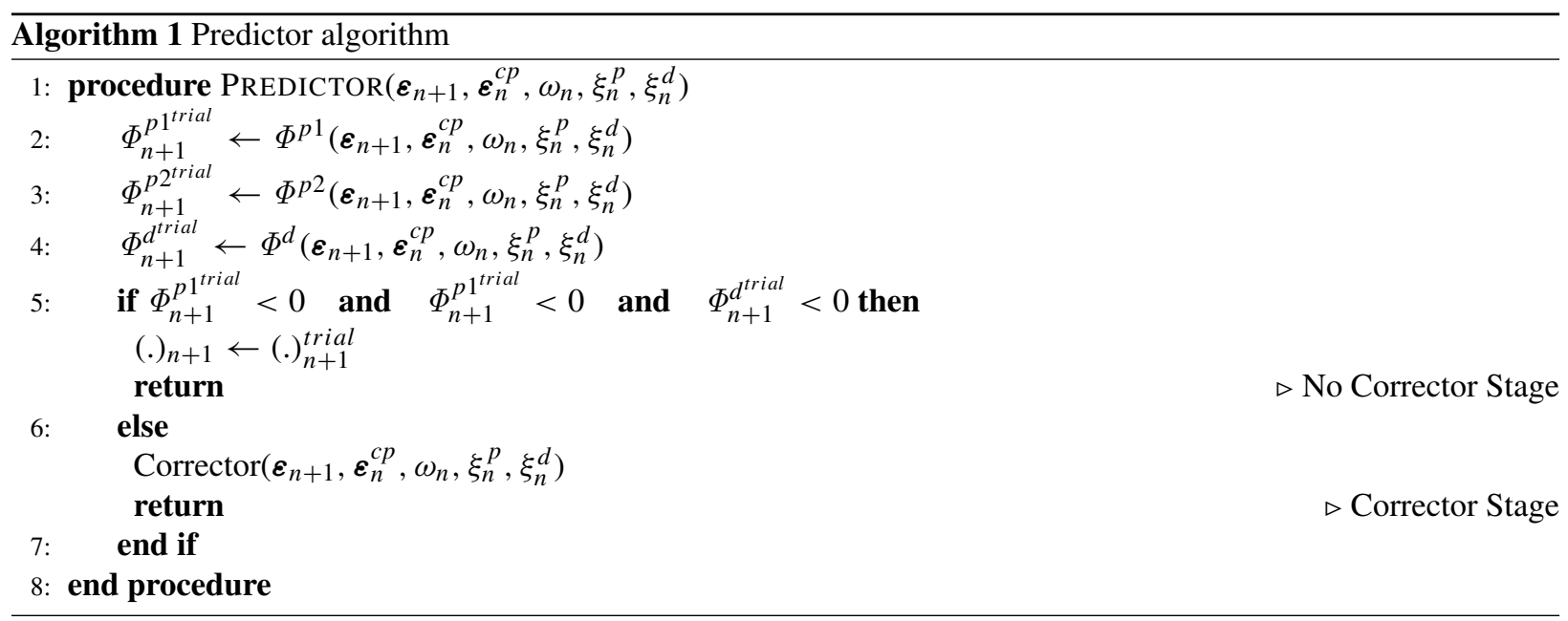

\section{Numerical examples}

The finite element code, implemented with the plastic damaging interphase model presented, has been applied to two numerical examples in order to test the numerical procedures and to show the capabilities of the model to simulate experimental evidences that in some cases cannot be reproduced by the classical ZTI model.

Numerical calculations have been carried out on two case-studies regarding masonry material specimens. The finite element models are two-dimensional in plane stress condition.

Example 1 consists of a masonry specimen subjected to a uniform uniaxial compression load. The aim of this analysis is to demonstrate the relevant role played by internal stresses in the initiation and evolution of damage within the mortar joints.

Example 2 regards the reproduction of the experimental results obtained by a diagonal compression test performed on a masonry specimen at the DICAM laboratory of material and structures. In this case the material property of the 


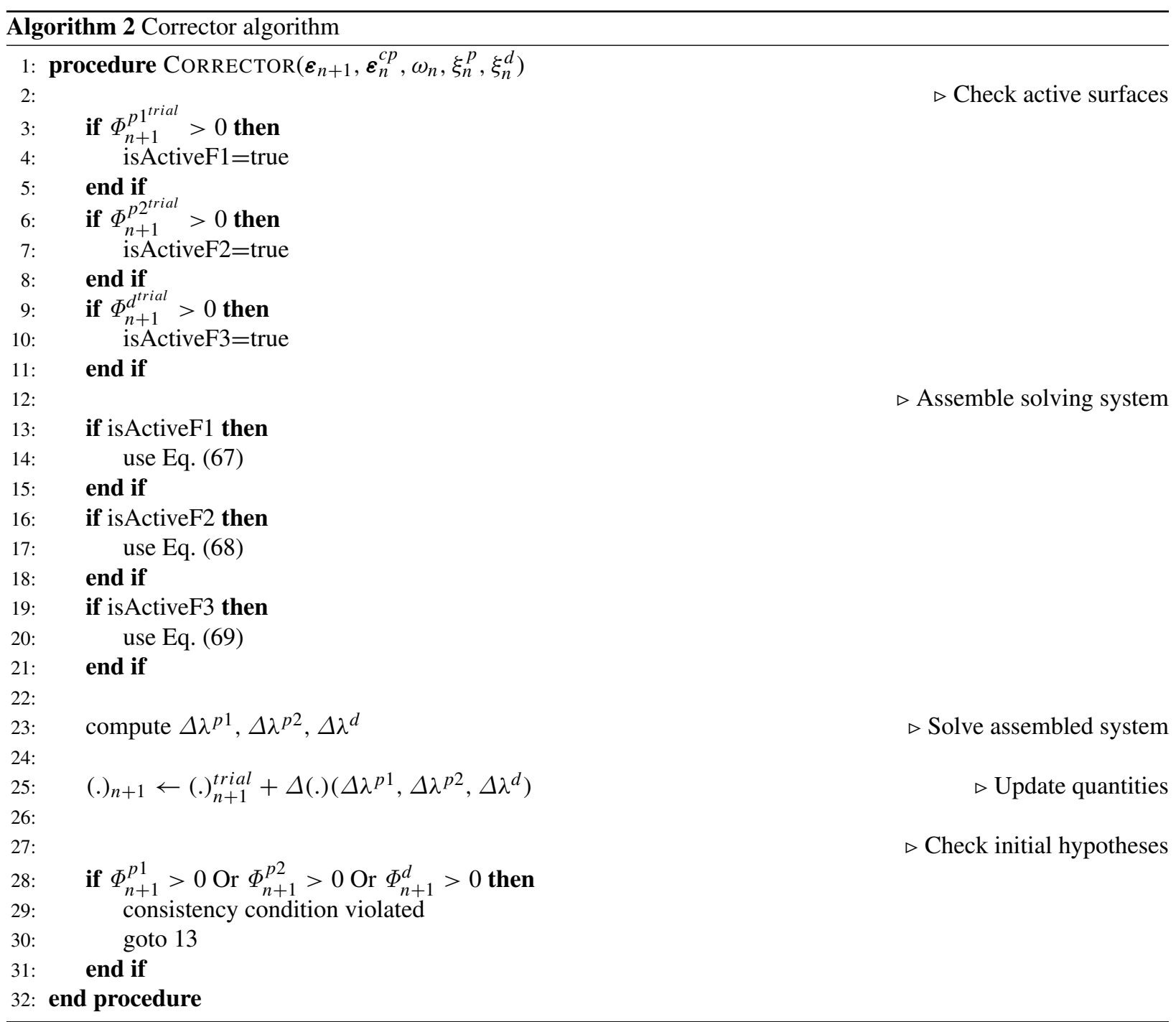

blocks and of the mortar are known because evaluated in separate tests and the interface properties are calibrated in order to fit the overall observed response of the material.

Displacement-driven analyses have been performed in order to capture the post-peak behavior.

\subsection{Example 1}

A masonry specimen constituted by a brick-mortar-brick sequence is uniformly compressed and the initiation and evolution of damage is studied for the case of mortar joint stiffer than bricks. In this condition, the bricks tend to expand more than the mortar in the horizontal direction because of the Poisson effect, but due to the higher stiffness, the mortar opposes to this deformation. The bricks are, therefore, subjected to compression stresses while the mortar to tension along the horizontal axis.

The geometry of the specimen, loads and displacement boundary conditions are illustrated in Fig. 3. The model depth is posed equal to $120 \mathrm{~mm}$.

The basic load $q=2[\mathrm{kN} / \mathrm{mm}]$ is monotonically increased with the load multiplayer $\alpha$. The elastic parameters adopted for the brick and mortar materials and plastic and damage parameters of the interphase used are reported in Tables 1 and 2, respectively.

The difference between the elastic moduli is exasperated to test the numerical performance of the interphase element in the non linear range and to show more clearly the damage of the joint. 


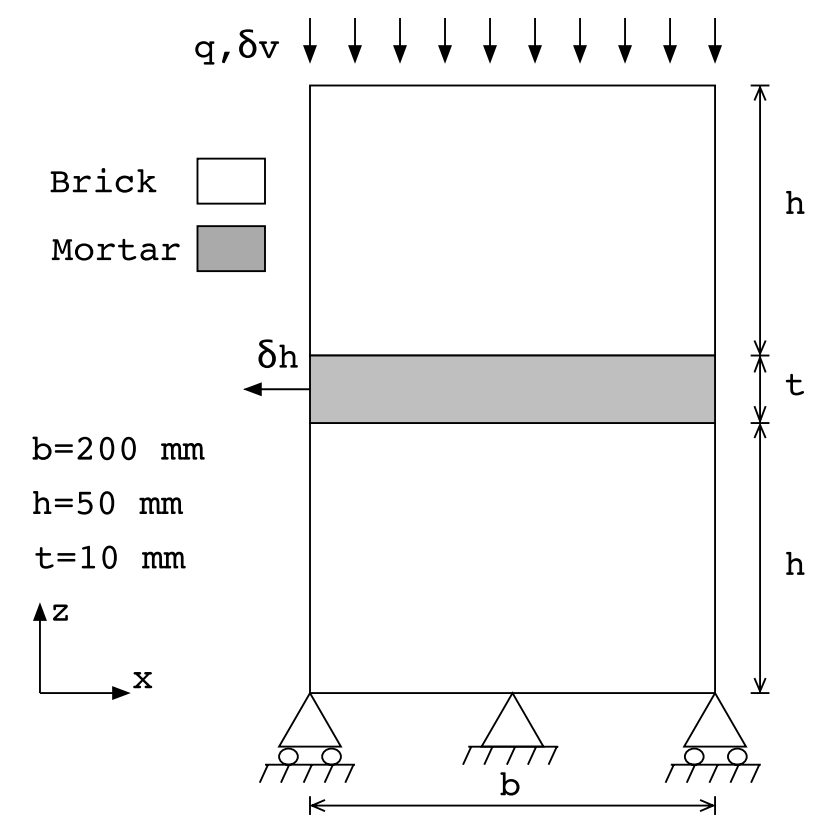

Fig. 3. Example 1, masonry specimen subjected to uniaxial compression test.

Table 1

Example 1, Elastic parameters.

\begin{tabular}{lll}
\hline & Bricks & Mortar joints \\
\hline $\mathrm{E}[\mathrm{MPa}]$ & 500 & 15,000 \\
$v$ & 0.3 & 0.2 \\
\hline
\end{tabular}

Table 2

Example 1, Interphase plastic and damage parameters.

\begin{tabular}{lllllll}
\hline$\sigma_{0}[\mathrm{MPa}]$ & $c_{0}[\mathrm{MPa}]$ & $\phi\left[{ }^{\circ}\right]$ & $\delta\left[^{\circ}\right]$ & $h_{p}$ & $\zeta_{0}$ & $h_{d}$ \\
\hline 1.0 & 4.5 & 30 & 20 & 1.0 & 0.001 & 0.0025 \\
\hline
\end{tabular}

The numerical experiment is carried out making use of a finite element model with a refined mesh constituted by $20 \times 80$ two-dimensional continuum elements for each brick and 80 interphase elements simulating the mortar joint.

In Fig. 4 the numerical results are illustrated in terms of internal stress $\sigma_{x}$ and normal and tangential contact tractions $\sigma_{z}, \tau$.

In particular in Fig. 4(a), the distribution of the interphase stresses is reported for an elastic load step. According to the theoretical treatment of the problem, the mortar joint is uniformly compressed in the vertical direction and is subjected to a tensile internal stress with a parabolic type profile starting from the zero value at the border and attaining the maximum value in the middle. The profile of the tangential stress is anti-symmetric with a zero value in the middle and at the borders.

The first fracture appears in the middle of the interphase due to the damage of the bulk material for a load multiplier $\alpha=0.22$. From the observation of the internal stress profile it is clear that the fracture is formed along the thickness of the joint, subdividing the interphase into two equal parts. A marked modification of the tangential traction $\tau$ distribution and a localized disturb in the normal traction profile can be also noticed.

For a value of $\alpha=0.35$, other two fractures appear in the two parts of the joint saving the symmetry of the problem. The stress profiles are reported in the same Fig. 4(c). Increasing the compression load, Fig. 4(d), it can be observed a sort of periodic onset of other fractures and asymptotically the interphase degenerates into the ZTI. 

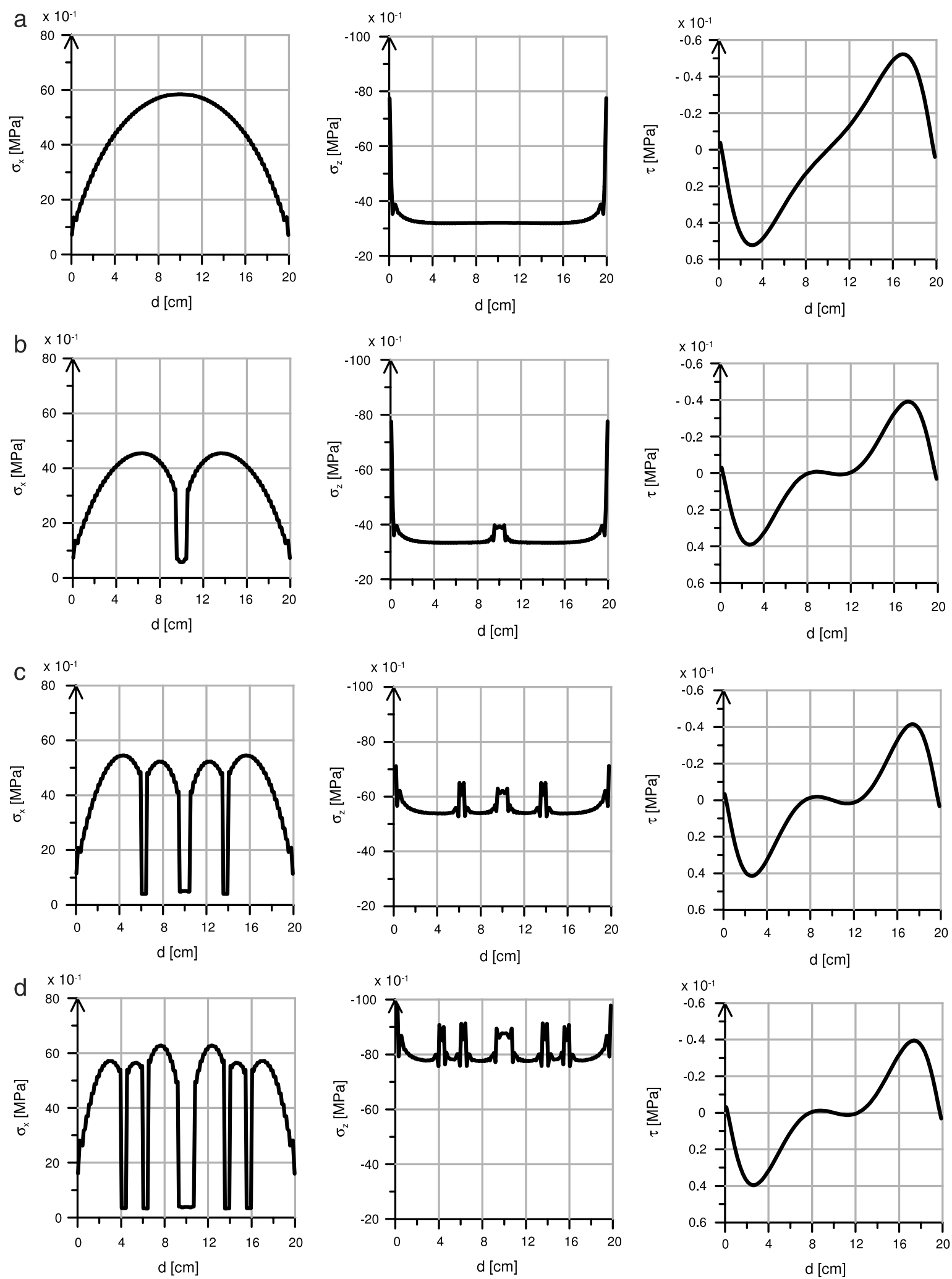

Fig. 4. Example 1, internal and contact stresses in the joint for different values of load multiplier: $\alpha=0.21$ (a), 0.22 (b), $0.35,0.50$ (d). 

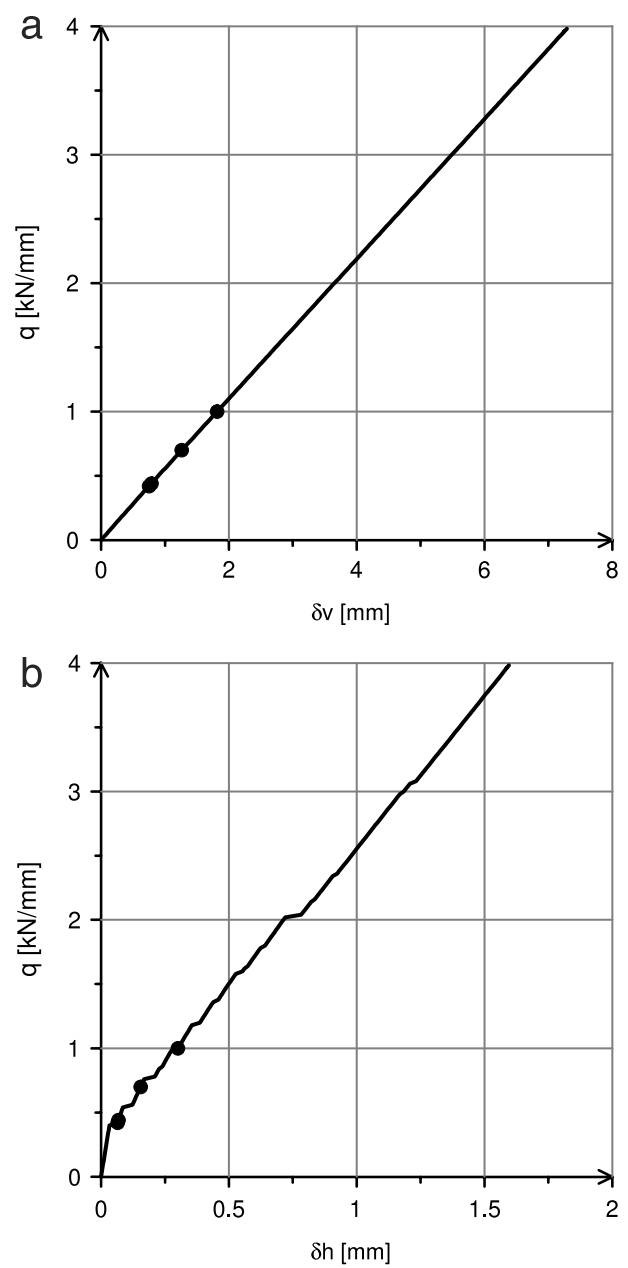

Fig. 5. Example 1, load-displacement diagrams.

The overall response of the masonry specimen is represented in the load-displacement diagrams of Fig. 5. As expected, the masonry specimen in the direction orthogonal to the joint does not show the effects of damage initiation and evolution. On the other hand, the fractures formation is evident when the load multiplier is plotted against the horizontal displacement of the joint border since the diagram has a saw-tooth shape. In particular, the first fracture appearing in the middle of the joint determines a quite evident reduction of the overall stiffness.

\subsection{Example 2}

The diagonal compression test performed on masonry specimens is used to determine the tensile strength of the composite material. A square masonry panel is subjected to a compressive load directed along one diagonal of the panel and the collapse mechanism generally observed is characterized by a crack starting from the center of the specimen and passing through mortar joints. The standard interpretation of the test is that a diagonal crack appears when the tensile principal stress in the material, assumed homogeneous, attains its maximum value.

In this numerical example a diagonal compression test developed at the DICAM laboratory is simulated. The masonry specimen is made up of four courses of sandstone blocks assembled with calcium-cement mortar, Fig. 6. The specimen has a squared shape with a length of $67 \mathrm{~cm}$ for each side. The single block is $33 \mathrm{~cm}$ wide, $16 \mathrm{~cm}$ high and $19.5 \mathrm{~cm}$ thick. The mortar layer has a thickness of $1 \mathrm{~cm}$.

During the compression tests the load $P$ has been monotonically increased up to the limit load value corresponding to the collapse of the panel. The relative displacements along the $y$ direction between the points $\mathrm{A}, \mathrm{B}$ and along the $x$ direction between the points $\mathrm{C}, \mathrm{D}$ have been registered in both sides of the specimen. 


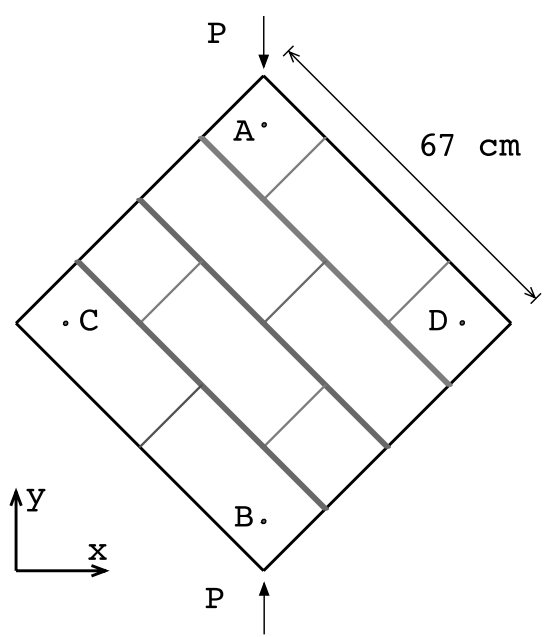

Fig. 6. Example 2, masonry specimen subjected to diagonal compression test.

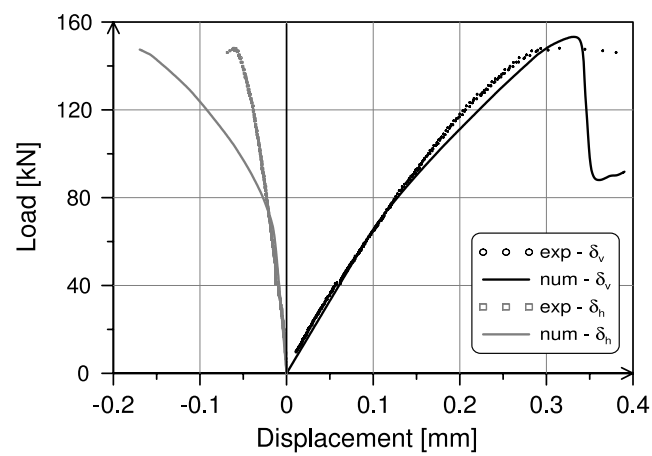

Fig. 7. Example 2, experimental and numerical load-displacement diagrams.

Table 3

Example 2, Elastic parameters.

\begin{tabular}{lll}
\hline & Bricks & Mortar joints \\
\hline E [MPa] & 11,142 & 37,000 \\
$v$ & 0.25 & 0.14 \\
\hline
\end{tabular}

The experimental points are plotted in Fig. 7 in terms of applied load $P$ and relative displacements $\delta_{h}=u_{C x}-u_{D x}$ and $\delta_{v}=u_{B y}-u_{A y}$. The limit load, assumed equal to the peak value, is 148,07 kN corresponding to the vertical and horizontal relative displacements $\delta_{h}=0.07 \mathrm{~mm}$ and $\delta_{v}=0.40 \mathrm{~mm}$, respectively. From the crack path experimentally obtained and depicted in Fig. 8, it appears that the fracture prevalently follows the mortar joints and passes through the first block of the second course.

Assuming the masonry material isotropic and making use of the elastic solution provided by Frocht [19], the diagonal tensile strength of the masonry material results $f_{d t}=P_{u} / 2 A=0.57 \mathrm{MPa}$ where $A$ is the area of the panel cross section.

The virtual diagonal compression test is performed by a finite element model where the blocks are considered linear elastic and modeled making use of two-dimensional continuum elements (4-noded) and the mortar joints are simulated by elastoplastic-damaging interphase elements (4-noded). The single block is discretized by $4 \times 8$ two-dimensional elements and the single mortar joint by 8 interphase elements.

The elastic properties of block and mortar materials are reported in Table 3 . The inelastic parameters of the interphase are reported in Table 4. 
a

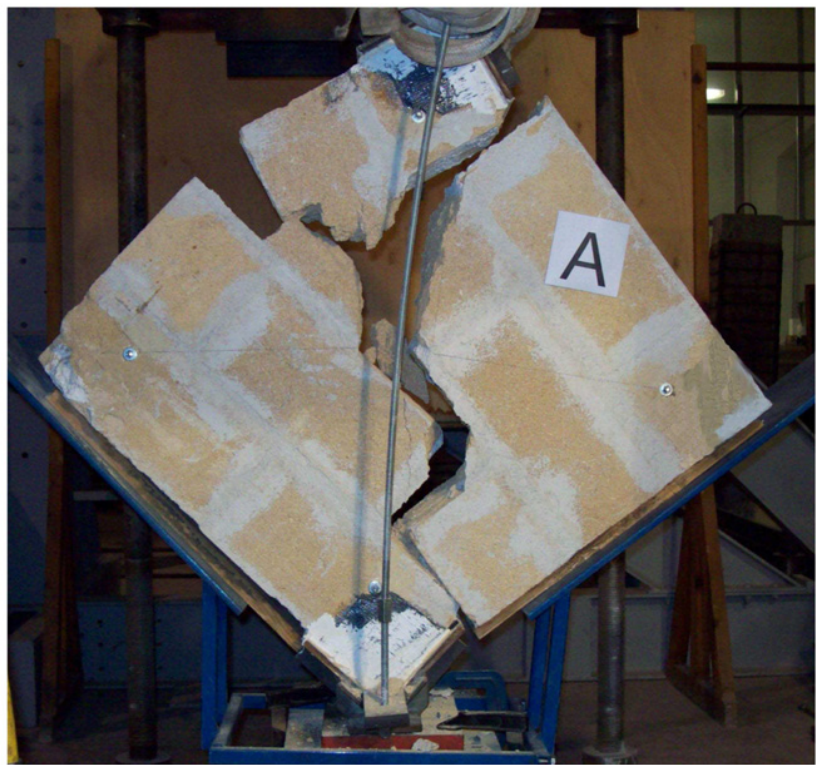

b

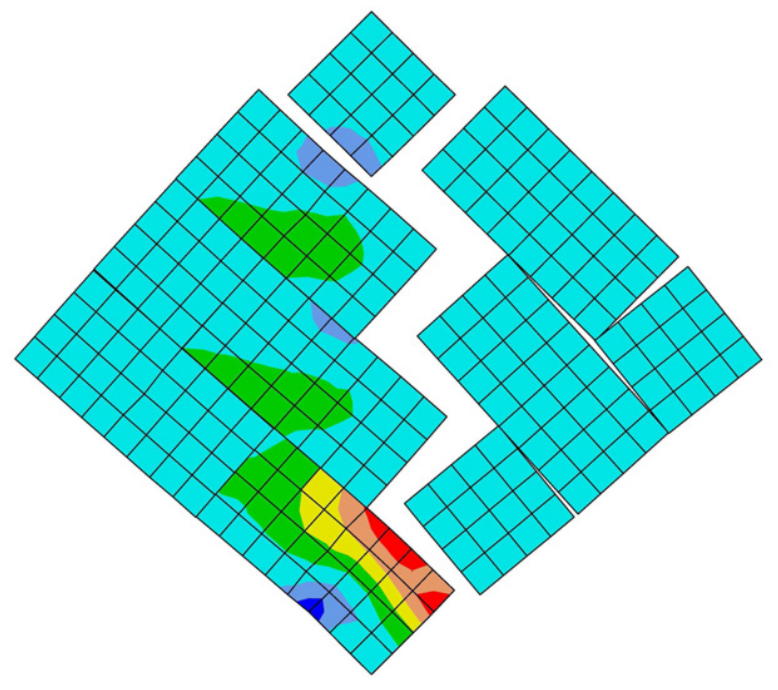

PRIN. STRESS 1

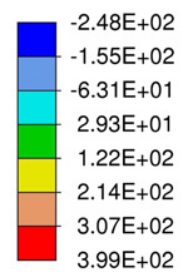

Current View

$\operatorname{Min}=-2.48 \mathrm{E}+02$

$X=-8.75 E+00$

$\mathrm{Y}=-3.86 \mathrm{E}+01$

$\operatorname{Max}=3.99 \mathrm{E}+02$

$\mathrm{X}=2.51 \mathrm{E}+00$

Time $=4.00 \mathrm{E}+02$

Fig. 8. Example 2, experimental and numerical, collapse mechanism.

Table 4

Example 2, Interphase plastic and damage parameters.

\begin{tabular}{lllllll}
\hline$\sigma_{0}[\mathrm{MPa}]$ & $c_{0}[\mathrm{MPa}]$ & $\phi\left[^{\circ}\right]$ & $\delta\left[^{\circ}\right]$ & $h_{p}$ & $\zeta_{0}$ & $h_{d}$ \\
\hline 1.0 & 5.0 & 35 & 20 & 0.04 & 0.00001 & 0.001 \\
\hline
\end{tabular}

The numerical results in terms of applied load and relative displacements are illustrated in the diagram of Fig. 7 together with the experimental points. In Fig. 8 the numerical and collapse mechanisms are illustrated. In the numerical model, the main fracture is forced to follow a zig-zag pattern since the masonry blocks are indefinitely elastic and this fact leads to some discrepancy between the numerical and experimental results. Overall, the virtual test well reproduces the experimental one. 


\section{Conclusions}

The mesomodeling of masonry structures is treated in the present paper making use of the interphase concept to simulate the mechanical behavior of mortar joints. The interphase model represents the enhancement of the zerothickness interface and is particularly useful for those cases where the joint internal stresses and strains play a crucial role in the response of the composite material.

It has been shown a peculiar feature of the interphase model. Differently to the ZTI model, where the non linear phenomena in the thin layer and at the interfaces are both governed by the contact tractions and a unique constitutive law must describe the overall response, the distinction of contact tractions and internal stresses allows to introduce different failure conditions for the physical interfaces and for the bulk material. In the present work it has been assumed that the joint stiffness degradation is related to the damage of the bulk material and that the onset of irreversible displacements coincides with the attainment of a specific yield condition at the physical interfaces.

On the base of the previous assumptions, the non linear interphase model has been formulated straightforwardly from an appropriate definition of the Helmholtz free energy where contact and internal strains represent the kinematical variables and where internal variables are introduced in order to regulate the evolution of stiffness degradation and of irreversible displacements.

The non linear interphase constitutive laws have been rewritten in discrete form suitable to be implemented in a standard finite element code. The principal aspects of the algorithm implementation are illustrated in detail and the effectiveness of the numerical procedure has been validated with two examples.

The numerical results show that the interphase model is capable to simulate the non linear deformation phenomena at the joints and the failure modes of the masonry material observed in the experimental tests, which cannot be predicted with the standard zero thickness interface models. Furthermore, the numerical values match quite well with the experimental measurements.

\section{Acknowledgments}

The authors acknowledge the financial support given by the Italian Ministry of Education, University and Research (MIUR) under the PRIN09 project 2009XWLFKW-005, A multiscale approach for the analysis of decohesion processes and fracture propagation.

\section{References}

[1] A. Corigliano, O. Allix, Some aspects of interlaminar degradation in composites, Comput. Methods Appl. Mech. Engrg. 185 (2000) $203-224$.

[2] G. Alfano, M.A. Crisfield, Finite element interface models for the delamination analysis of laminated composite: mechanical and computational issues, Internat. J. Numer. Methods Engrg. 50 (2001) 1701-1736.

[3] K. William, I. Rhee, B. Shing, Interface damage model for thermomechanical degradation of heterogeneous materials, Comput. Methods Appl. Mech. Engrg. 193 (2004) 3327-3350.

[4] T. Stankowski, K. Runesson, S. Sture, Fracture and slip of interfaces in cementitious composites, J. Eng. Mech. (ASCE) 119 (2) (1993) 292-314.

[5] H.R. Lofti, P.B. Shing, Interface model applied to fracture of masonry structures, J. Struct. Eng. (ASCE) 120 (1) (1994) 63-80.

[6] P.B. Lourenco, J.G. Rots, Multisurface interface model for analysis of masonry structures, J. Eng. Mech. (ASCE) 123 (7) (1997) 660-668.

[7] G. Giambanco, S. Rizzo, R. Spallino, Numerical analysis of masonry structures via interface models, Comput. Methods Appl. Mech. Engrg. 190 (2001) 6493-6511.

[8] G. Giambanco, G. Fileccia Scimemi, Mixed mode failure analysis of bonded joints with rate-dependent interface models, Internat. J. Numer Methods Engrg. 67 (2006) 1160-1192.

[9] G. Alfano, E. Sacco, Combining interface damage and friction in a cohesive-zone model, Internat. J. Numer. Methods Engrg. 68 (2006) $542-582$

[10] F. Parrinello, B. Failla, G. Borino, Cohesive-frictional interface constitutive model, Int. J. Solids Struct. 46 (2009) $2680-2692$.

[11] A. Spada, G. Giambanco, P. Rizzo, Damage and plasticity at the interfaces in composite materials and structures, Comput. Methods Appl. Mech. Engrg. 198 (2009) 3884-3901.

[12] E. Sacco, F. Lebon, A damage-friction interface model derived from micromechanical approach, Int. J. Solids Struct. 49 (26) (2012) $3666-3680$.

[13] G. Giambanco, Z. Mroz, The interphase model for the analysis of joints in rock masses and masonry structures, Meccanica 36 (1) (2001) $111-130$.

[14] G. Giambanco, G. Fileccia Scimemi, A. Spada, The interphase element, Comput. Mech. 50 (3) (2012) 353-366.

[15] R.H. Atkinson, B.P. Amadei, S. Saeb, S. Sture, Response of masonry bed joints in direct shear, J. Struct. Eng. (ASCE) 115 (9) (1989) 2276-2296. 
[16] W.T. Koiter, Stress-strain relations uniqueness and variational problem for elasto-plastic materials with a singular yield surface, Quart. Appl. Math. 11 (1953) 350-354.

[17] J.C. Simo, J.G. Kennedy, S. Govindjee, Non-smooth multisurface plasticity and viscoplasticity. Loading/unloading conditions and numerical algorithms, Internat. J. Numer. Methods Engrg. 26 (1988) 2161-2185.

[18] A. Anthoine, Homogenization of periodic masonry: plane stress, generalized plane strain or 3D modeling, Commun. Numer. Methods Eng. 13 (1997) 319-326.

[19] M.M. Frocht, Recent advances in photoelasticity, ASME Trans. 55 (1931) 52-73. 